\title{
Recent results on analytical plasma turbulence theory: Realizability, intermittency, submarginal turbulence, and self-organized criticality
}

\author{
J A Krommes \\ Princeton University, P. O. Box 451, Princeton, NJ 08543-0451 USA
}

\begin{abstract}
Recent results and future challenges in the systematic analytical description of plasma turbulence are described. First, the importance of statistical realizability is stressed, and the development and successes of the Realizable Markovian Closure are briefly reviewed. Next, submarginal turbulence (linearly stable but nonlinearly self-sustained fluctuations) is considered and the relevance of nonlinear instability in neutral-fluid shear flows to submarginal turbulence in magnetized plasmas is discussed. For the Hasegawa-Wakatani equations, a self-consistency loop that leads to steady-state vortex regeneration in the presence of dissipation is demonstrated and a partial unification of recent work of Drake (for plasmas) and of Waleffe (for neutral fluids) is given. Brief remarks are made on the difficulties facing a quantitatively accurate statistical description of submarginal turbulence. Finally, possible connections between intermittency, submarginal turbulence, and selforganized criticality (SOC) are considered and outstanding questions are identified.
\end{abstract}

PACS numbers: 52.35.Ra, 47.20.Ky

Submitted to: Plasma Phys. Control. Fusion

\section{Introduction}

Two fundamental challenges in the systematic analytical theory of plasma turbulence are to (i) extract statistical information from the Klimontovich or Vlasov equation (or their gyrokinetic analogs) for the microscopic density $\widetilde{f}$, where the electric field $\widetilde{\boldsymbol{E}}$ is treated as random; (ii) exhibit and analyze detailed dynamical mechanisms that give rise to the statistics. In this paper I attempt to put into perspective some recent results on (i), and I describe some new calculations that bear on (ii).

Statistical information can be gathered either through the values of moments or cumulants (useful for simple descriptions of transport processes and fluctuation spectra) or through the shape of entire probability density functions (PDF's) (useful for highly intermittent and non-Gaussian processes). The moment description leads one immediately to the hierarchal structure that the $n$-point cumulants (such as $f \doteq$ $\langle\widetilde{f}\rangle$ for $n=1$, where $\doteq$ denotes definition and $\langle\ldots\rangle$ denotes an ensemble average) 
are driven by $(n+1)$-point cumulants (such as $\langle\delta \boldsymbol{E} \delta f\rangle$ for $n+1=2$, where $\delta f \doteq \widetilde{f}-f)$. This gives rise to the most familiar form of the statistical closure problem [Kraichnan (1959); Kraichnan (1961); Krommes (1984)], which is how to relate high-order cumulants to lower-order ones. Attempts to find closed equations for restricted (single-point, say) PDF's lead to even more fundamental closure difficulties [Chen, Chen and Kraichnan (1989)].

In plasma physics, most attention has been paid to moment closures. Physical reasoning based on natural extensions of perturbation theory [Drummond and Ross (1973)] was reasonably successful in identifying the natural building blocks of a complete theory of strong plasma turbulence, such as waves, particles, and non-wave-like phase-space granulations (clumps or holes) [Dupree (1969); Dupree (1972)]. However, attempts to systematize these notions with formal mathematics encountered signficant difficulties. Dupree (1966) discussed an elaborate test-wave formalism, but workable truncations of the general equations turn out to be at best appropriate for problems of passive advection (statistically specified random fields), not for the practically important self-consistent problems in which the fields are determined from $\tilde{f}$ via Poisson's equation. As a consequence, the resulting resonancebroadening theory [Dupree (1966); Dupree (1967); Dupree (1968)] does not conserve energy [Dupree and Tetreault (1978); Similon (1981); Krommes (1984)].

Superficially, the general philosophy of Dupree's approach appears to have much in common with Kraichnan's earlier direct-interaction approximation (DIA) [Kraichnan (1959)]. However, the derivation of the DIA is substantially more robust and systematic; the formalism conserves quadratic energy-like invariants and exhibits other important consistency properties. The DIA was proposed for Vlasov turbulence by Orszag and Kraichnan (1967), but their comprehensive work was substantially ignored for at least a decade, until the work of Montgomery (1977). However, gradually it was realized that the DIA provides not only a reasonable description of many aspects of plasma turbulence [DuBois and Espedal (1978)] but also a robust starting point for recovering some of the simpler, asystematic approximations such as resonancebroadening theory or clump theory [Krommes (1978)]. DuBois and Rose (1981) made detailed DIA calculations for Langmuir turbulence. In the context of fusion, Krommes (1982) showed in the context of a simple three-mode model that the DIA was capable of quantitatively predicting saturation levels of linearly unstable modes interacting through a Terry-Horton nonlinearity [Terry and Horton (1982)]. More detailed discussion and further references on statistical plasma physics were given by Krommes (1997b).

Key to the practical success of the DIA is its rigorous derivation from an underlying stochastic amplitude equation, the random-coupling model (RCM) [Kraichnan (1961); Orszag and Kraichnan (1967); Krommes (1996)]. The mere existence of such a stochastic model, and hence of a positive-definite probability density functional of the random amplitude, implies an infinite number of realizability constraints [Kraichnan (1980)] between cumulants of different orders. The significance of those constraints will be further discussed in the next section. 
Although the DIA is very useful theoretically, it is difficult to work with in highly anisotropic situations. It is possible to systematically develop Markovian approximations to the DIA; the most popular one is the eddy-damped quasi-normal Markovian (EDQNM) approximation [Orszag (1977)]. For incompressible NavierStokes turbulence, the second-order statistics predicted by that theory are statistically realizable. However, it was an unwelcome surprise to find that realizability fails when the EDQNM is applied to certain problems with linear waves [Bowman (1992); Bowman et al. (1993)]. Since such waves are ubiquitous in fusion and other plasma applications, it was urgent to develop a realizable generalization of the EDQNM. That was accomplished in the form of Bowman's Realizable Markovian Closure (RMC) [Bowman (1992); Bowman et al. (1993); Bowman and Krommes (1997)].

In Sec. 2 I will very briefly review the development and successes of the RMC. Although it likely does not represent the ultimate Markovian closure, it brings the theory of moment closure in plasmas to a relatively satisfactory plateau.

Current research focuses on a quantitatively detailed theory of submarginal turbulence -i.e., self-sustained turbulence that persists in the face of a linearly stable spectrum. Both dynamical mechanisms and their statistical description are of interest. This subject is in its infancy; some limited recent progress and promising lines of research are described in Sec. 3. The topic is not unrelated to certain ideas about self-organized criticality (SOC), also mentioned briefly in Sec. 3. The paper concludes with brief discussion in Sec. 4.

\section{Statistical realizability and the Realizable Markovian Closure}

The ultimate goal of a statistical theory is to predict the fully multivariate space-time probability density functional. In practical situations, that goal is unrealistic and one is content with reduced information such as low-order cumulants. Because PDF's are positive-definite, cumulants obey an infinite number of realizability inequalities. Most importantly, the covariance matrix must be positive-definite.

Unfortunately, statistical closures do not usually predict cumulants directly; instead, they assert time-evolution equations for them. Because those equations are nonlinear, it is not guaranteed that realizability inequalities remain satisfied for all times even if they are initially. A well-known counterexample is the quasinormal closure [Ogura (1963) and references therein], which can predict fluctuation levels that diverge toward $-\infty$ after one eddy-turnover time of an initially excited mode. This difficulty is well understood [Orszag (1977)] as the failure of the theory to take account of nonlinear scrambling, which provides a nonlinear correlation time that limits the effective temporal range of interaction. The renormalization inherent to the DIA cures that problem, yet it is not guaranteed on plausibility arguments alone that an arbitrary renormalization that merely includes a nonlinear correlation time will be well-behaved [Kraichnan (1961)] What makes the DIA special is the underlying random-coupling model. The secondorder statistics predicted by the DIA are the exact ones for the RCM, so they must 
obey the second-order realizability inequalities. Indeed, for statistics of any order one can define the DIA to be the statistical description of the RCM [Krommes (1996)].

Although the RCM is most fundamental, an alternative Langevin representation is frequently useful. That has been discovered not only for the DIA itself, but also for the EDQNM [Leith (1971); Kraichnan (1970)]. For the generic amplitude equation

$$
\left(\partial_{t}+i \Omega_{\boldsymbol{k}}\right) \psi_{\boldsymbol{k}}=\frac{1}{2} \sum_{\Delta} M_{\boldsymbol{k p q}} \psi_{\boldsymbol{p}}^{*} \psi_{\boldsymbol{q}}^{*},
$$

where $\Omega_{\boldsymbol{k}}$ is complex and $\Delta$ indicates all $\boldsymbol{p}, \boldsymbol{q}$ such that $\boldsymbol{k}+\boldsymbol{p}+\boldsymbol{q}=0$, second-order statistics of the EDQNM are predicted by the Langevin equation

$$
\left(\partial_{t}+i \Omega_{\boldsymbol{k}}+\widehat{\mu}_{\boldsymbol{k}}\right) \psi_{\boldsymbol{k}}(t)=\widetilde{f}_{\boldsymbol{k}}(t) \equiv \widetilde{w}(t) \sum_{\Delta} M_{\boldsymbol{k p q}} \sqrt{\theta_{\boldsymbol{k} \boldsymbol{p}}} \xi_{\boldsymbol{p}}^{*}(t) \xi_{\boldsymbol{q}}^{*}(t) .
$$

Here $\widehat{\mu}_{\boldsymbol{k}} \doteq-\sum_{\Delta} M_{\boldsymbol{k} \boldsymbol{q}} M_{p \boldsymbol{q} \boldsymbol{k}}^{*} \theta_{\boldsymbol{k} \boldsymbol{q}}^{*}\left\langle\left|\delta \psi_{\boldsymbol{q}}\right|^{2}\right\rangle, \widetilde{w}(t)$ is a Gaussian white-noise process of unit amplitude whose presence ensures the Markovian nature of the resulting approximation, and the auxiliary random field $\xi_{\boldsymbol{k}}$ is constrained to have variance identical to that of $\psi_{\boldsymbol{k}}$. The term $\sqrt{\theta}$ is necessary on dimensional grounds. Physically, $\theta_{\boldsymbol{k} \boldsymbol{p} \boldsymbol{q}}$ is the interaction time between the three directly interacting modes $(\boldsymbol{k}, \boldsymbol{p}, \boldsymbol{q})$ that form the triad $\boldsymbol{k}+\boldsymbol{p}+\boldsymbol{q}=0$. It obeys $\partial_{t} \theta_{\boldsymbol{k} \boldsymbol{p} \boldsymbol{q}}+\left(i \Omega_{\boldsymbol{k}}+\widehat{\mu}_{\boldsymbol{k}}+\right.$ c.p. $) \theta_{\boldsymbol{k p q}}=1$. Note that its dynamics involves the same linear and nonlinear physics as does $\psi_{\boldsymbol{k}}$ itself.

Actually, (2) is physically relevant only for $\operatorname{Re} \Omega_{\boldsymbol{k}}=0$. For $\operatorname{Re} \Omega_{\boldsymbol{k}} \neq 0$ (note that linear waves are ubiquitous in plasma physics), $\theta_{\boldsymbol{k} p \boldsymbol{q}}$ becomes complex and its interpretation as an interaction time is lost. It does not help to use only the real part of $\theta$ on the right-hand side of (2), since the real part of an oscillating complex amplitude can easily be negative. Negative interaction times make no physical sense, nor does the resulting imaginary noise $\widetilde{f}_{\boldsymbol{k}}$. If one ignores these difficulties and attempts to proceed, one finds [Bowman (1992); Bowman et al. (1993)], both numerically and analytically, that the complex-valued EDQNM can predict catastrophically negative intensities - the premier signature of nonrealizability.

A partial cure is to modify the statistical description of the transient dynamics in such a way that realizability is guaranteed. That was accomplished in Bowman's derivation of his Realizable Markovian Closure (RMC), a modification of the EDQNM that asserts a more symmetrical form of the fluctuation-dissipation ansatz, guarantees that $\operatorname{Re} \theta$ remains positive, and asymptotes in steady state to the predictions of the EDQNM. The theory has been explored for various few-mode models [Bowman et al. (1993)], the Hasegawa-Mima (HM) [Hasegawa and Mima (1978)] equation [Bowman and Krommes (1997)], and the Hasegawa-Wakatani (HW) model [Hu et al. (1995); Hu et al. (1997)]. Hu's work is particularly striking: for the HW model [Hasegawa and Wakatani (1983); see equations (3) below], he found excellent agreement between the predictions of the RMC for the particle flux and spectrum and the results of direct numerical simulation; see Fig. 10 of Hu et al. (1997). It is clear that statistical closure theory, much maligned historically, can make very successful predictions for turbulent transport if it is carefully and quantitatively analyzed. 
Key questions about the statistical dynamics of plasmas remain. A general one is the proper description of intermittency (highly non-Gaussian statistics). The work of $\mathrm{Hu}$ et al. (1997) shows that second-order closures such as the RMC can capture some important low-order statistical features (e.g., particle transport) of situations in which some fields (e.g., vorticity) are highly intermittent. However, it is a challenge to accurately predict high-order statistics and to understand their influence on low-order ones. Application of the DIA to statistics of any order is well-defined but can be shown to be inadequate in general [Chen, Herring, Kerr and Kraichnan (1989); Krommes (1996)]. A promising route is the theory of mapping closures [Chen, Chen and Kraichnan (1989); Das and Kaw (1995); Krommes (1997b)].

\section{Submarginal turbulence}

A standard paradigm for turbulence that was used in the interpretation [Hu et al. (1997)] of the turbulent-transport calculations described above invokes linear instability (growth rate $\gamma_{\boldsymbol{k}}$ ) to drive up fluctuations at forcing wave numbers $k_{f}$. Those fluctuations are then transferred to other wave numbers by nonlinear mode coupling and are ultimately dissipated at dissipation wave numbers $k_{d}$. In this scenario, the fluctuation level of the resulting forced, dissipative steady state would scale with (some power of) the linear growth rate and would vanish as $\gamma_{\boldsymbol{k}} \rightarrow 0^{+}$. The transition to turbulence occurs via a supercritical bifurcation. An early analysis of such a bifurcation for collisional drift waves was done by Hinton and Horton (1971).

In the context of neutral-fluid dynamics governed by the Navier-Stokes equation, it has been long known that the standard paradigm fails in various important practical situations involving shear flows. Linear stability analysis of such examples as planar Couette flow or pipe Hagen-Poiseuille flow show that such flows are stable to infinitesimal perturbations at all Reynolds numbers $R$ (so the linear-instability threshold is $\left.R_{l}=\infty\right)$, yet in reality turbulence is observed for $R$ greater than a relatively small and finite critical Reynolds number $R_{c}$. Such flows must therefore be unstable to perturbations of finite amplitude. On the other hand, it can be shown [Joseph (1976)] that there exists an energy-stability threshold $R_{e}$ below which perturbations of arbitrary amplitude decay monotonically. In general, then, one must have $R_{e} \leq R_{c} \leq R_{l}$. If $R_{c}$ is strictly less than $R_{l}$, then for $R_{c}<R<R_{l}$ one can have subcritical bifurcations and the possibility of submarginal turbulence - self-sustained random fluctuations that persist in the face of complete linear stability. In recent fluid simulations of plasmas, submarginal turbulence has been observed by Waltz (1985), Scott (1992), Drake et al. (1995), and Itoh et al. (1996), among others. Extensive earlier work on nonlinear instability in Vlasov plasmas [cf. Berman et al. (1983) and references therein] is relevant but cannot be discussed here. 


\subsection{Dynamical mechanisms for submarginal turbulence}

Key challenges in the theory of submarginal turbulence are to predict the value of $R_{c}$, to describe a detailed dynamical mechanism for self-sustainment, and to marry such a mechanism with a consistent statistical closure. These goals are far from being achieved; however, some partial results are known. An important technical theorem is that submarginal turbulence is possible only if the linear evolution operator $\mathrm{L}$ (where $\partial_{t} \psi=\mathbf{L} \psi+$ n.l. terms) is non-normal $\ddagger$ [Henningstone and Reddy (1994)]. Such operators can transiently amplify initial perturbations to very large levels, even though they must ultimately decay if the spectrum is stable. This observation led to discussion of an "almost-linear" mechanism for self-sustainment that combined non-normal transient amplification with a particular kind of nonlinear feedback [Trefethen et al. (1993); Baggett et al. (1995)]. However, Waleffe (1995b) has challenged the general applicability of this mechanism for physical shear flows and has proposed an alternate mechanism [Waleffe (1997)] to be discussed further below.

Linear non-normality is necessary, if not sufficient. One immediate conclusion [Schekochihin (1998)] is that single-field models of the HM variety, of the general form (1) and where the nonlinear term conserves an appropriate energy, cannot support submarginal turbulence. An equivalent statement is that for such models the energystability threshold coincides with the threshold for linear instability. This can be demonstrated by a simple variational calculation.

Although this negative result about single-field models might appear to be trivial, it has profound consequences. In the context of a statistical description of the turbulent state, it implies that internal nonlinear noise [cf. the right-hand side of (2)] cannot, in and of itself, be the underlying mechanism for submarginal turbulence, as seems to be suggested in some of the literature [Terry and Diamond (1984)]. The statistical descriptions of all energy-conserving dynamical models include nonlinear noise, yet not all of them can support submarginal turbulence. One must look deeper for the mechanism of self-sustainment.

Non-normality appears to be generic for multiple-field models of plasma fluctuations driven by profile gradients. A specific example is the two-field HW system

$$
\begin{aligned}
& \left(\partial_{t}+\boldsymbol{V}_{E} \cdot \nabla\right) \omega=-D_{\|} \partial_{z}^{2}(\varphi-n)+\mu \nabla_{\perp}^{2} \omega, \\
& \left(\partial_{t}+\boldsymbol{V}_{E} \cdot \nabla\right) n=-D_{\|} \partial_{z}^{2}(\varphi-n)-\kappa \partial_{y} \varphi+D \nabla_{\perp}^{2} n,
\end{aligned}
$$

where $\varphi$ is the electrostatic potential, $\omega \doteq \nabla_{\perp}^{2} \varphi$ is the $z$ component of the vorticity of the $\boldsymbol{E} \times \boldsymbol{B}$ flow $\boldsymbol{V}_{E} \doteq \hat{\boldsymbol{z}} \times \nabla \varphi, n \doteq N-\langle N\rangle$ is the fluctuation in the electron density, the $D_{\|}$terms incorporate the effects of resistive dissipation, and $\kappa \doteq-\nabla \ln \langle N\rangle$. The linear matrix of Eqs. (3) is non-normal due to the presence of the diamagnetic term in the density equation. For such systems it is clear that submarginal turbulence, if it exists,

$\ddagger$ A non-normal matrix is one whose eigenvectors are not orthogonal. An example is $L=\left(\begin{array}{ll}\alpha & 0 \\ \kappa & \beta\end{array}\right)$. The eigenvalues $\alpha$ and $\beta$ are stable for negative $\alpha$ and $\beta$, yet $\exp (\mathrm{L} t)$ exhibits transient amplification even for distinct $\alpha$ and $\beta$. In plasmas, $\kappa$ represents the diamagnetic effects involving profile gradients. 
must be driven by the profile gradients (the origin of non-normality). But it is the mere presence of the gradients, not linear instability, that is essential.

In neutral-fluid shear flows, non-normality is associated with gradients of the laminar streamwise flow velocity $U$. For such flows, Waleffe has argued in convincing detail [Waleffe (1995a); Waleffe (1997)] for a generic three-part mechanism for self-sustainment, involving (i) advection of mean shear by weak streamwise rolls (vortices); (ii) linear instability of the resulting streaks (spanwise velocity fluctuations); (iii) nonlinear self-interaction of the streaks that re-energizes the original streamwise rolls. Note that linear instability is possible here because the perturbations are made not around the background profile, which is linearly stable, but around the rolls that are self-consistently shown to be maintained at a finite level. That non-normality is associated with background gradients in both the plasma and neutral-fluid cases leads one to inquire whether key elements of Waleffe's mechanism should apply to plasmas as well, even though the detailed linear dynamics may appear to be quite different in the two cases. Although much work remains undone, the preliminary answer is yes.

In particular, for a natural generalization of the HW system (that included soundwave propagation), Drake et al. (1995) have proposed the following self-sustainment mechanism: (i) generation of a sheared radial§ flow by a $\left(k_{y} \neq 0, k_{z}=0\right)$ mode; (ii) driftwave instability (with $k_{z} \neq 0$ ) driven by the $\partial n / \partial y$ resulting from the radial flow; (iii) nonlinear self-interaction of the drift waves leading to regeneration of the $k_{z}=0$ mode. If one identifies the streamwise direction with the direction of the magnetic field, $\|$ the mechanisms sound broadly similar. In both, it is a $k_{\|}=0$ mode that is selfsustained, where $\|$ refers to the streamwise or magnetic-field direction. The physics of the $k_{\|} \neq 0$ linear instabilities that grow on the $k_{\|}=0$ background differ in detail (wakelike in the neutral fluid, drift-wave-like in the plasma), but that may not be important to the ultimate regeneration.

By analyzing a low-dimensional model that omitted perpendicular collisional dissipation (therefore working in a non-stationary, large-amplitude limit), Drake et al. found a nonlinear instability that supported the physical regeneration mechanism sketched above. It should be noted that the mere presence of such an instability does not distinguish the type of underlying bifurcation, which could be either supercritical or subcritical. A modest contribution was made by Krommes and Boldyrev (1996), who included dissipation in Drake's model, analyzed the bifurcation structure in detail, and

$\S$ Note that the directions in items (i) and (ii) are rotated by $90^{\circ}$ from the frequently discussed sheared poloidal flow $\left(k_{y}=0, k_{z}=0\right)$ and conventional drift waves excited by $x$-directed gradients of the background profile.

$\|$ It is an unfortunate annoyance that the standard choice of coordinate systems differs in the neutral-fluid and plasma literature. For neutral-fluid shear flows, the choice is $(x, y, z)_{\text {fluid }}=$ (streamwise, background gradient, spanwise). For slab approximations to toroidal magnetized plasmas, one writes instead $(x, y, z)_{\text {plasma }}=$ (background gradient, poloidal, toroidal). One proceeds from the neutral-fluid to the equivalent plasma by cyclically reducing the fluid coordinates by one: $(x, y, z)_{\text {fluid }} \rightarrow$ $(z, x, y)_{\text {plasma. }}$ Waleffe's streamwise velocity $U(y, z)$ corresponds to plasma density perturbations $\bar{N}(x, y)$ with $k_{z}=0$. 
found the expected subcritical bifurcation.

A much more refined calculation presently underway [Son (1998)] aspires to raise the mathematical description of the submarginal plasma dynamics to the level of Waleffe's calculations; the HW equations are appropriately illustrative. Ultimately the analysis must be conducted in a sheared magnetic field (probably with the more complete equations discussed by Drake et al.) in order to ensure linear stability, but that formidable calculation has not yet been done. Nevertheless, useful insight can be extracted from a shear-free model. I merely sketch the analysis here; details will be presented elsewhere.

Because drift-wave fluctuations are driven by density gradients, consider the density equation $(3 b)$ written for total density $N$ :

$$
\partial_{t} N(x, y, z)+\boldsymbol{V}_{E} \cdot \nabla N=-D_{\|} \partial_{z}^{2}(\varphi-n)+D \nabla_{\perp}^{2} N .
$$

One may work on the interval $x \in(-1,1)$ and impose the boundary conditions $N(1)=0$, $N(-1)=2, V_{E, x}( \pm 1)=V_{E, y}( \pm 1)=0$. The $-D_{\|} \partial_{z}^{2}$ term is required for linear instability; however, fluctuations with $k_{z}=0$ are essential for the nonlinear self-sustainment mechanism. Therefore, temporarily ignore any $z$-dependent portions of the vorticity field $\omega$ or $\boldsymbol{E} \times \boldsymbol{B}$ flow; I will show that a $z$-independent contribution to $\omega$ can be selfconsistently regenerated. Then upon averaging (4) over $z$ (denoted by an overline), one obtains

$$
\partial_{t} \bar{N}(x, y)+\overline{\boldsymbol{V}}_{E} \cdot \nabla \bar{N}=D \nabla_{\perp}^{2} \bar{N}
$$

-i.e., $\bar{N}$ obeys a passive advection-diffusion equation. In the spirit of a self-sustaining mechanism, one may seek stationary solutions of (5).

To proceed, one needs an informed guess about the underlying vorticity field. The $z$ average of $(3 a)$ leads to

$$
\partial_{t} \bar{\omega}(x, y)+\overline{\boldsymbol{V}_{E} \cdot \nabla \omega}=\mu \nabla_{\perp}^{2} \bar{\omega} .
$$

(The overline has been kept outside of the $\boldsymbol{V}_{E}$ for later use.) At sufficiently low Reynolds number, as a first guess it is not unreasonable to ignore the nonlinearity and to build the base flow from the eigenvalue problem $\nabla_{\perp}^{2} \omega=\lambda \omega, \omega=\nabla_{\perp}^{2} \varphi$. A (no-slip) solution is readily obtained:

$$
\varphi=-\left[\frac{\cos (p x)}{\cos p}-\frac{\cosh (\gamma x)}{\cosh (\gamma)}\right]\left[\frac{\sin (\gamma y)}{\gamma}\right],
$$

where $p \tan p+\gamma \tanh \gamma=0$. For this flow, a steady-state solution of (5) is shown in Fig. 1a. One sees the development of significant perturbations in the $y$ direction. In the fluid context, these spanwise fluctuations are called streaks.

If sufficiently large, the density perturbations $\bar{N}(x, y)$ can be unstable to drift waves (with nonzero $k_{z}$ ) propagating in the $x$ direction. Numerical spectral analysis of the linearization of Eqs. (3) around $\bar{N}(x, y)$ and the assumed initial vorticity field is straightforward with the aid of Chebyshev-Fourier expansion. Until shear is included, it is not possible to discuss the ultimate effect. However, as a preliminary exercise, one 


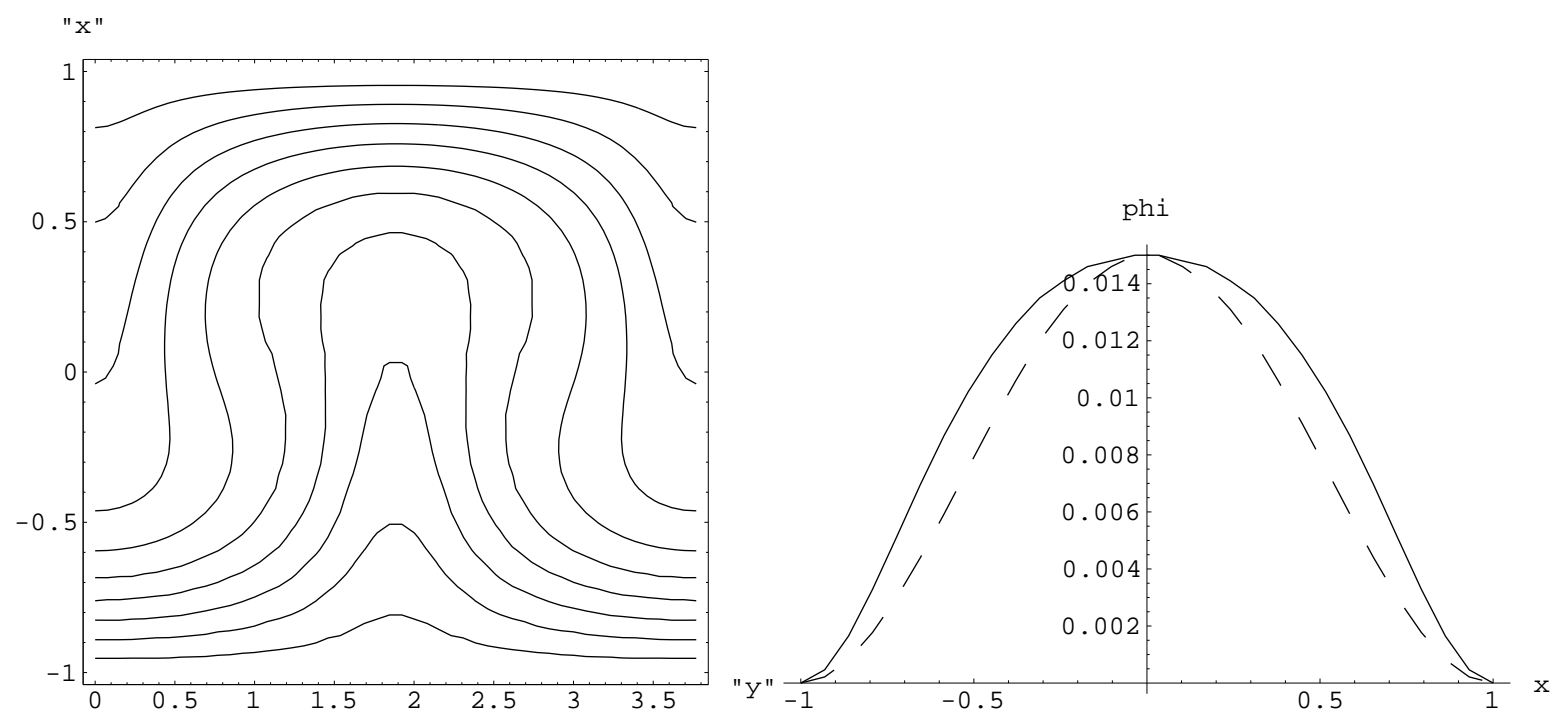

Figure 1. (a) Solution of the steady-state advection-diffusion equation for $V_{0}=-0.025, R_{D}=400$, $\gamma=5 / 3$. (b) Solid line: steady-state solution of (6) with nonlinear forcing determined by the most unstable eigenfunction. Dashed line: original potential, formula (7).

can make some contact with the work of Drake et al. by replacing in the diamagnetic term of the linearized density equation the $\bar{N}(x, y)$ that follows from solution of the advection-diffusion equation by its $x$ average, $\bar{N}(y)$. This eliminates the standard driftwave instability driven by $\partial_{x} \bar{N}$. A preliminary, low-resolution calculation of the resulting growth rate has been performed; an instability is still obtained. Partly on the basis of the physical arguments presented by Drake et al., it is expected that this instability persists in the presence of magnetic shear. The key idea is to linearize around a self-consistently generated "streaky" state rather than around a fluctuation-free equilibrium.

The final stage of the proposed self-sustainment mechanism is to re-energize the assumed $k_{z}=0$ vortices. That comes from the $\overline{\boldsymbol{V}_{E} \cdot \nabla \omega}$ term in (6), which in steady state is a Poisson equation for $\omega$ that ultimately determines $\varphi$. The $x$ dependence of the solution of that equation, with the most unstable eigenfunction from the spectral calculation inserted as a quasilinear forcing, is shown in Fig. 1b for the fundamental $y$ mode. The shape of the driven solution is in quite reasonable agreement with the initial assumption (7). (Since the amplitude of the marginal eigenfunction is undetermined from the linear spectral calculation, it has been normalized such that the driven solution matches the initial potential at $x=0$.) Thus, a plausible mechanism for self-sustainment has been demonstrated.

It must be stressed that the present work represents technically a very minor modification of Waleffe's extensive pioneering efforts. Its conceptual importance is that the basic nonlinear regeneration mechanism appears to be upheld in this plasma model even though the details of linear instability differ drastically between the neutral-fluid shear flows and the magnetized plasma. Thus, the present analysis supports Waleffe's belief that the mechanism he investigated is generic and robust. 
The present work should be compared with that of Drake et al. Whereas those authors ignored explicit perpendicular dissipation and concentrated on the timedependent development of the nonlinear instability, the present calculation attempts to find a self-consistent forced, dissipative steady state; it can be viewed as a generalization of the work of Krommes and Boldyrev (1996).

Much remains to be done. In the absence of shear, it is feasible [Son (1998)] to extract from the HW equations a low-order dynamical system that exhibits the principal self-sustainment loop; this is an energy-conserving, dissipative generalization of Drake's model that will be described elsewhere. Preliminary numerical investigations show that chaotic behavior akin to the Lorenz strange attractor ensues for some parameter regimes. Quantitative calculations in the presence of shear are daunting, but well-defined. Work on both of these topics is in progress.

\subsection{Statistical closures and submarginal turbulence}

There is ultimately no substitute for a detailed dynamical description of submarginal self-sustainment (or of any other turbulent state). However, to the extent that the result is steady-state turbulence, coarse-grained statistical descriptions are also relevant and may ultimately be simpler, as they focus on average intensities and are not forced to deal with complicated, microscopic phase information. Unfortunately, the ability of conventional closures such as the DIA to faithfully represent interesting bifurcation scenarios remains only poorly understood.

In the context of thermal convection, Kraichnan (1964) noted that the DIA likely predicted a bifurcation between a static $(\omega=0)$ steady state and a fluctuating one, the latter being stable for sufficiently large Prandtl number. Similar observations were made by Herring (1969). On the other hand, McLaughlin (1974) applied the DIA to the famous Lorenz equations, known to exhibit a strange attractor for certain parameters whose onset is subcritical [McLaughlin and Martin (1975)]; he concluded that the DIA was not particularly faithful and did not recognize the subcritical bifurcation at all.

Technically, the problem is that the nonlinear structure of the DIA and similar closures is more complicated than that of the original primitive dynamics, so is difficult to analyze. One might expect, however, that a closure that properly captures gross dynamical properties of the physical state, including linear non-normality, energy conservation, and sensible mode coupling between $k_{\|}=0$ and $k_{\|} \neq 0$ modes, could in principle suffice to predict submarginal turbulent intensities. In that spirit, Schekochihin and Krommes (1997) have analyzed the structure of the DIA for a truncated HW model with just three planes of constant $k_{\|}$: one for $k_{\|}=0$; the other two describing complex conjugates of a typical $k_{\|} \neq 0$ mode. Many $\boldsymbol{k}_{\perp}$ 's are allowed on each plane. The virtue of this calculation is that it is readily possible to demonstrate approximations that retain nonlinear noise and properly conserve energy. Preliminary calculations suggest that energy-conserving statistical models can be built that are compatible with the scenarios of Scott (1992), Biskamp and Zeiler (1995), and Drake et al. (1995). Further discussion 
will be presented elsewhere.

A more heuristic approach to the statistical description of submarginal turbulence has been taken by K. Itoh, S. Itoh, and coworkers in a series of detailed papers, representative ones of which include Itoh et al. (1994) and Itoh et al. (1996). They use a Dupree-style Markovian renormalization in which the effects of turbulence are represented by eddy-viscosity-like transport coefficients added to linear theory. The dependence of those coefficients on fluctuation intensity is obtained through a series of additional technical approximations that can be understood as a severe reduction of the EDQNM in which the symmetry of the triad interaction time $\theta_{\boldsymbol{k p q}}$ is lost and

off-diagonal terms are ignored. Nonlinear noise $\left[\tilde{f}_{\boldsymbol{k}}\right.$ in $\left.(2)\right]$ is neglected, so analysis of the steady state reduces to study of a nonlinear dispersion relation. Subcritical solutions are predicted for several multi-field models (including the HW system, according to recent unpublished work by the author). In some cases, those appear to be in tantalizing agreement with numerical simulations [Itoh et al. (1996)]. Such an approach, founded on the structure of linear theory, at least qualitatively captures the requisite nonnormality. On the other hand, the neglect of nonlinear noise is a significant omission that would seem to vitiate any quantitative predictions, as its effect enters at the same order as the eddy viscosities. Furthermore, in some single-field model problems [Krommes and Boldyrev (1996)] the bifurcation properties of the intensity vs gradient curve are strongly influenced by the presence or absence of noise: intensity bifurcations that are subcritical in the unjustified absence of noise become supercritical in its presence. (Recall that single-field models can exhibit only supercritical excitation.) Nevertheless, a speculation is that if the actual dynamics exhibit submarginal turbulence and if that is predicted by a sensible noise-free closure, as Itoh et al. find, then the proper nature of the bifurcation may be preserved by the addition of nonlinear noise. Having positive-definite covariance, that noise will tend to raise the predicted steadystate fluctuation level, but need not alter the qualitative submarginal dependence of intensity on parameters. A systematic and quantitative calculation of a nontrivial multifield model is sorely needed here; such research is in progress [Son (1998)].

\subsection{Submarginal turbulence and self-organized criticality}

Recently it has been suggested that ideas of self-organized criticality (SOC) may be relevant to problems of turbulent transport in plasmas [Diamond and Hahm (1995)]. The basic philosophy and mathematics underlying what is conventionally called SOC have been well spelled out in the original references [Bak et al. (1987); Bak et al. (1988)] and in more popular accounts [Bak and Tang (1989); Bak (1996)]. Some recent plasmaphysics-oriented discussion was given by Dendy and Helander (1997). Briefly, it is shown that simple discrete automata that implement particle-conserving toppling rules with nonlinear thresholds can exhibit avalanches at all scales, and that such systems when driven with random additive forcing can achieve steady states with submarginal profiles; this is the link to the work of the last several subsections. It is argued that the long- 
ranged correlations exhibited in such systems may be somehow relevant to observable tokamak transport phenomena.

It is intriguing that such simple models can exhibit submarginal behavior, but one should be very cautious about generalizing to the real types of multidimensional, partial-differential advection-diffusion equations encountered in practice. Krommes (1997a) has emphasized that in practice the proper forcing is multiplicative (e.g., random $\boldsymbol{E} \times \boldsymbol{B}$ drifts), not additive as in a conventional sandpile model. The spacetime correlation properties of the random coefficient are crucial in determining the ultimate dynamical behavior. In particular, a coefficient effectively white in time gives rise to a standard diffusion equation with entirely local transport. To the extent that the diffusion coefficient depends on space, the solutions to the transport equation subject to macroscopic boundary conditions may be unusual, yet would (or should) not be invoked as evidence of SOC.

The key to the global physics is thus the dynamical (or statistical) properties of the advecting field. Within the context of a simple stochastic model, Krommes (1997a) showed that with some effort it is possible to arrange those properties such that submarginal profiles result over at least part of the domain. This was done, essentially, by building in the answer in the form of a subcritical bifurcation for the turbulent intensity; no first-principles dynamical calculation was done. The significance of that calculation is therefore not its dynamical fidelity, which is minimal, but rather that it shows that there need be no link between submarginal profiles and discrete or longranged transport events; the latter were absent in the model described by Krommes (1997a).

On the other hand, low-order dynamical models of submarginal self-sustainment can exhibit intermittency that can lead to anomalous scaling and may be related to the transport events discussed in the SOC literature. However, whether or not the discrete models of SOC live in the same universality class as continuum models with $\boldsymbol{E} \times \boldsymbol{B}$ nonlinearity is still an open question. A proper answer requires a much deeper understanding of the nonlinear dynamics and self-sustainment mechanisms of continuum models such as described in the last section. Considerable further work is required.

\section{Discussion}

I have briefly discussed various topics of current interest in basic nonlinear plasma turbulence theory. A recent success was the development of a realizable Markovian statistical closure, the RMC; excellent agreement was found between its predictions and direct numerical simulation. Current research focuses on the dynamical mechanisms for submarginal turbulence and the extent to which that phenomenon is correctly handled in statistical theory. The outline of a proper theory is beginning to emerge, although quantitative calculations are very difficult. The interplay between submarginal turbulence, intermittency, and self-organized criticality is also of great interest, although there are very few solid results as yet. All in all, the systematic mathematical description 
of random nonlinear phenomena in plasmas is slowly yielding to detailed analysis, but still presents major and enticing challenges.

\section{Acknowledgments}

Aspects of the work reported here were performed in collaboration with S. Boldyrev, J. Bowman, G. Hu, M. Ottaviani, A. Schekochihin, and S. Son. I enjoyed detailed discussions of this research with J. Burkholder; P. Holmes offered useful suggestions. The research was supported by U.S.D.o.E. Contract No. DE-AC02-76-CHO-3073.

\section{References}

Baggett J S, Driscoll T A and Trefethen L N 1995 Phys. Fluids 7, 833.

Bak P 1996 How Nature Works: The Science of Self-Organized Criticality Copernicus New York.

Bak P and Tang C 1989 Phys. Today 42 (1), S27.

Bak P, Tang C and Wiesenfeld K 1987 Phys. Rev. Lett. 59, 381.

Bak P, Tang C and Wiesenfeld K 1988 Phys. Rev. A 38, 364.

Berman R H, Tetreault D J and Dupree T H 1983 Phys. Fluids 26, 2437.

Biskamp D and Zeiler A 1995 Phys. Rev. Lett. 74, 706.

Bowman J C 1992 Realizable Markovian Statistical Closures: General Theory and Application to Drift-wave Turbulence PhD thesis Princeton University.

Bowman J C and Krommes J A 1997 Phys. Plasmas 4, 3895.

Bowman J, Krommes J A and Ottaviani M 1993 Phys. Fluids B 5, 3558.

Chen H D, Chen S and Kraichnan R H 1989 Phys. Rev. Lett. 63, 2657.

Chen H, Herring J R, Kerr R M and Kraichnan R H 1989 Phys. Fluids A 1, 1844.

Das A and Kaw P 1995 Phys. Plasmas 2, 1497.

Dendy R O and Helander P 1997 Plasma Phys. Control. Fusion 39, 1947.

Diamond P H and Hahm T S 1995 Phys. Plasmas 2, 3640.

Drake J F, Zeiler A and Biskamp D 1995 Phys. Rev. Lett. 75, 4222.

Drummond W E and Ross D W 1973 in B. E Keen and E. W Laing, eds, 'Turbulence and Non-linear Effects in Plasmas' U.K.A.E.A. Culham Laboratory Abingdon, England p. 1.

DuBois D and Espedal M 1978 Plasma Phys. 20, 1209.

DuBois D F and Rose H A 1981 Phys. Rev. A 24, 1476.

Dupree T H 1966 Phys. Fluids 9, 1773.

Dupree T H 1967 Phys. Fluids 10, 1049.

Dupree T H 1968 Phys. Fluids 11, 2680.

Dupree T H 1969 in J Fox, ed., 'Turbulence of Fluids and Plasmas' Polytechnic Press Brooklyn, New York p. 3.

Dupree T H 1972 Comm. Plasma Phys. and Controlled Fusion 1, 33.

Dupree T H and Tetreault D J 1978 Phys. Fluids 21, 425.

Hasegawa A and Mima K 1978 Phys. Fluids 12, 87.

Hasegawa A and Wakatani M 1983 Phys. Rev. Lett. 50, 682.

Henningstone D S and Reddy S C 1994 Phys. Fluids 6, 1396.

Herring J R 1969 Phys. Fluids 12, 39.

Hinton F L and Horton, Jr. C W 1971 Phys. Fluids 14, 116.

Hu G, Krommes J A and Bowman J C 1995 Phys. Lett. A 202, 117.

Hu G, Krommes J A and Bowman J C 1997 Phys. Plasmas 4, 2116.

Itoh K, Itoh S I, Fukuyama A, Yagi M and Azumi M 1994 Plasma Phys. Control. Fusion 36, 279.

Itoh K, Itoh S I, Yagi M and Fukuyama A 1996 J. Phys. Soc. Jpn. 65, 2749. 
Joseph D D 1976 Stability of Fluid Motions I Springer Berlin.

Kraichnan R H 1959 J. Fluid Mech. 5, 497.

Kraichnan R H 1961 J. Math. Phys. 2, 124. Erratum: J. Math. Phys. 3, 205 (1962).

Kraichnan R H 1964 Phys. Fluids 7, 1163.

Kraichnan R H 1970 J. Fluid Mech. 41, 189.

Kraichnan R H 1980 in R. H. G Helleman, ed., 'Nonlinear Dynamics' New York Academy of Sciences New York p. 37.

Krommes J A 1978 in 'Theoretical and Computational Plasma Physics' International Atomic Energy Agency Vienna p. 405.

Krommes J A 1982 Phys. Fluids 25, 1393.

Krommes J A 1984 in A. A Galeev and R. N Sudan, eds, 'Handbook of Plasma Physics' Vol. 2 NorthHolland Amsterdam chapter 5.5, p. 183.

Krommes J A 1996 Phys. Rev. E 53, 4865.

Krommes J A 1997 a Phys. Plasmas 4, 1342.

Krommes J A $1997 b$ Phys. Rep. 283, 5.

Krommes J A and Boldyrev S 1996 'Theoretical approaches to turbulent transport near marginal stability'. International Sherwood Fusion Theory Conf., abstract 1B01, unpublished.

Leith C E 1971 J. Atm. Sci. 28, 145.

McLaughlin J B 1974 The Transition to Turbulence in a Statically Stressed Fluid System PhD thesis Harvard University.

McLaughlin J B and Martin P C 1975 Phys. Rev. A 12, 186.

Montgomery D 1977 Proc. Indian Acad. Sci. 8A, 87.

Ogura Y 1963 J. Fluid Mech. 16, 33.

Orszag S A 1977 in R Balian and J.-L Peube, eds, 'Fluid Dynamics' Gordon and Breach New York p. 235.

Orszag S A and Kraichnan R H 1967 Phys. Fluids 10, 1720.

Schekochihin A A 1998. Private communication.

Schekochihin A A and Krommes J A 1997 Bull. Am. Phys. Soc. 42, 2036.

Scott B D 1992 Phys. Fluids B 4, 2468.

Similon P 1981 Renormalized Theory of Drift Wave Turbulence in Sheared Magnetic Fields PhD thesis Princeton University.

Son S 1998. Private communication.

Terry P and Horton W 1982 Phys. Fluids 25, 491.

Terry P W and Diamond P H 1984 in C. W Horton, Jr. and L. E Reichl, eds, 'Statistical Physics and Chaos in Fusion Plasmas' Wiley New York p. 335.

Trefethen L N, Trefethen A E, Reddy S C and Driscoll T A 1993 Science 261, 578.

Waleffe F 1995a Stud. Appl. Math. 95, 1.

Waleffe F $1995 b$ Phys. Fluids 7, 3060.

Waleffe F 1997 Phys. Fluids 9, 883.

Waltz R E 1985 Phys. Rev. Lett. 55, 1098. 